\title{
Patient Experiences of Neurofeedback for Chemotherapy- induced Peripheral Neuropathy: A Case Series Comparing Real and Placebo Treatment
}

\author{
Prinsloo S, ${ }^{1,2 *}$, Vallone $V^{1}$, Moreno $\mathbf{N}^{1}$, Sanchez $\mathbf{H}^{1}$, \\ Burgess $\mathrm{H}^{1}$, Ike $\mathrm{S}^{1}$, Novy $\mathrm{D}^{3}$, Barcenas $\mathrm{C}^{4}, \mathrm{Li} \mathrm{Y}^{5}$, \\ Lopez $\mathbf{G}^{\mathbf{1}}$, Bruera $\mathrm{E}^{\mathbf{1}}$ and Cohen $\mathrm{L}^{\mathbf{1}}$ \\ ${ }^{1}$ Department of Palliative, The University of Texas MD \\ Anderson Cancer Center, USA \\ ${ }^{2}$ Department of Neurosurgery, The University of Texas \\ MD Anderson Cancer Center, USA \\ ${ }^{3}$ Department of Pain Medicine, The University of Texas \\ MD Anderson Cancer Center, USA \\ ${ }^{4}$ Department of Breast Medical Oncology, The University \\ of Texas MD Anderson Cancer Center, USA \\ ${ }^{5}$ Department of Biostatistics, The University of Texas MD \\ Anderson Cancer Center, USA \\ *Correspondling author: Sarah Prinsloo, Department \\ of Neurosurgery, Department of Palliative, The University \\ of Texas MD Anderson Cancer Center, Rehabilitation, \\ and Integrative Medicine, 1515 Holcombe Boulevard, Unit \\ 1414, FCT 5.6006, Houston, TX, USA
}

Received: February 09, 2021; Accepted: February 24, 2021; Published: March 03, 2021

\begin{abstract}
Background: A major concern in interventional studies is the inability to accurately link patient report with objective measures. In this study, we associated functional brain measures with self-reported pain after patients underwent a neuromodulatory intervention. Specifically, Chemotherapy-Induced Peripheral Neuropathy (CIPN) adversely affects many cancer patients but few effective treatment options are available, and mechanisms are not well understood.
\end{abstract}

Objectives: We present three representative cases from a doubleblind, randomized, placebo-controlled trial which examined the efficacy of a targeted therapy of Electroencephalogram (EEG) Neurofeedback (NFB), in attenuating symptoms of CIPN. The primary outcome of the trial was efficacy of neurofeedback versus control groups. In this case series we explore mechanism, by linking patient reported outcomes with objective measures.

Methods: Symptom descriptions and EEG data were collected for patients enrolled in neurofeedback, placebo feedback, and waitlist conditions. Subjective pain ratings and EEG data were compared before and after the 10-week intervention.

Results: A patient receiving neurofeedback demonstrated decreased beta oscillations in Brodmann Area 6 (BA6) and reported noticeable decreases in numbness and temperature sensitivity. A patient receiving placebo demonstrated increased beta in BA6 and increased alpha oscillations in Brodmann areas 3 and 7 with improvement of symptoms. A waitlist participant showed no change in BA6 and reported increased neuropathic symptoms while on waitlist but subsequently received NFB treatment and reported symptom improvement.

Conclusions: This case series indicates that NFB may be used to achieve targeted reduction in beta oscillations to treat CIPN. Possible mechanisms of action and implications for CIPN treatment are discussed.

Keywords: Cancer pain; Chemotherapy-induced peripheral neuropathy; Neurofeedback; Neuromodulation; Electroencephalogram (EEG); Cancer survivorship

\section{Introduction}

Advances in cancer detection and treatment have resulted in improved survival rates and increased life expectancy across a wide array of cancers [1]. Chemotherapy-Induced Peripheral Neuropathy (CIPN) affects the majority of cancer patients (68\%) who undergo chemotherapy, with $60 \%$ continuing to report symptoms 3 months after treatment and $30 \%$ reporting symptoms 6 or more months later [2].

CIPN presents most commonly in the hands and feet, though sensations can extend beyond these areas. Symptoms can include pain, numbness, tingling, itching, cramping, difficulty perceiving temperature, and intolerance to heat or cold. Activities of daily living such as walking, bathing, washing dishes, and carrying items can become more difficult and potentially dangerous for those with CIPN.

Neurofeedback (NFB) is a non-invasive and non-pharmacological neuromodulatory intervention shown to be effective in the treatment of CIPN and other pain syndroms [3]. NFB is a variant of biofeedback that uses a brain-computer interface to modify patients' neuronal frequencies in real-time through operant conditioning. By increasing neuroplasticity over time, NFB is used to correct brainwave abnormalities associated with pain, such as excessive beta.

\section{Methods}

This research was approved by The University of Texas MD Anderson Cancer Center institutional review board. Patients experiencing CIPN at least 3 months after the end of chemotherapy signed an informed consent, provided baseline measures, and were randomly assigned to either NFB, Placebo Neurofeedback (PL), or a Waitlist (WL) group. Participants in the NFB group received rewards when they decreased beta activity. The PL group received rewards based on the same training parameters as NFB, however,
Austin Neurosurg Open Access - Volume 7 Issue 1 - 2021 Submit your Manuscript | www.austinpublishinggroup.com Prinsloo et al. @ All rights are reserved
Citation: Prinsloo S, Vallone V, Moreno N, Sanchez H, Burgess H, Ike S, et al. Patient Experiences of Neurofeedback for Chemotherapy-induced Peripheral Neuropathy: A Case Series Comparing Real and Placebo Treatment. Austin Neurosurg Open Access. 2021; 7(1): 1063 
their rewards were non-contingent and delivered according to a prerecorded EEG of an unrelated individual at rest.

Participants in NFB and PL received 20 sessions of their respective treatments and rated their neuropathic symptoms before and after each session. Over the same period, WL were queried 20 times for their neuropathy ratings. Patients and researchers were blinded to whether patients received NFB or PL and EEGs were recorded both pre- and post-treatment for all groups. Following completion of data collection, PL and WL participants were offered 20 sessions of NFB. To observe brain changes, each patient served as their own control.

\section{Results}

All three patients received surgery, a taxane-based chemotherapy, and radiation. For these cases, no adverse events were reported.

\section{Case 1: Neurofeedback treatment}

A 68-year-old female diagnosed with Stage III breast cancer and at consent reported experiencing CIPN for 9.7 years, which she rated as a 1/10 in her hands and a 3/10 in her feet, with 10 being the most severe. Her symptoms included profound numbness, which put her at risk for burns and other injuries due to decreased ability to sense temperature. Additionally, she reported an impaired sense of balance, and she had experienced two "face-first" falls prior to her participation in the study. The patient reported taking $50 \mathrm{mg}$ of Tramadol as needed in addition to $100 \mathrm{mg}$ of Gabapentin once a day. The patient experienced a noticeable decrease in foot numbness during the first NFB session and reported being able to feel her toes for the first time in years. Before treatment, she frequently found it necessary to warm her feet through external means (i.e., hot water bottle) due to cold intolerance. By session 3, she reported that her feet no longer needed to be heated. After 10 sessions, the patient stopped taking Gabapentin, yet her symptoms continued to improve. After 20 sessions, the patient reported improved gait, stable walking, the absence of falls, and decreased numbness in her toes compared to baseline. She rated the neuropathy in both her hands and feet as a $0 / 10$ post-treatment. The patient described her response to NFB as "a complete home run" and was particularly appreciative of her gains given that she lives alone. Her post-treatment EEG showed a decrease in beta frequencies (Figure 1).

\section{Case 2: Placebo treatment followed by elective NFB treatments}

A 61-year-old female diagnosed with Stage II breast cancer experiencing CIPN for 2 years at the time of consent rated her neuropathy as an 8/10 in both her hands and feet. She reported severe numbness and tingling in hands and feet that interfered

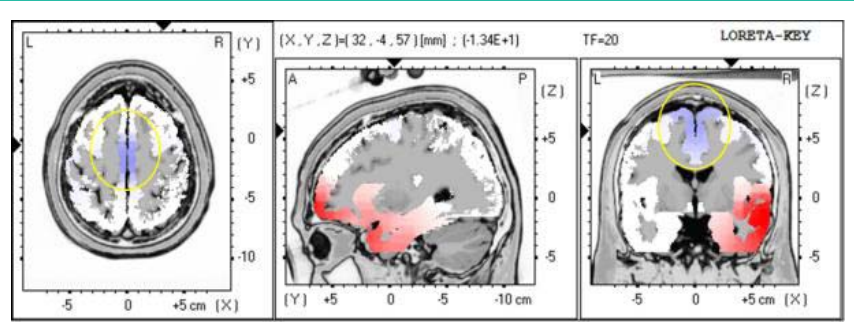

Figure 1: Neurofeedback: Image shows decreases in Beta $(20 \mathrm{HZ})$ as calculated by post treatment minus pre-treatment in BA6.
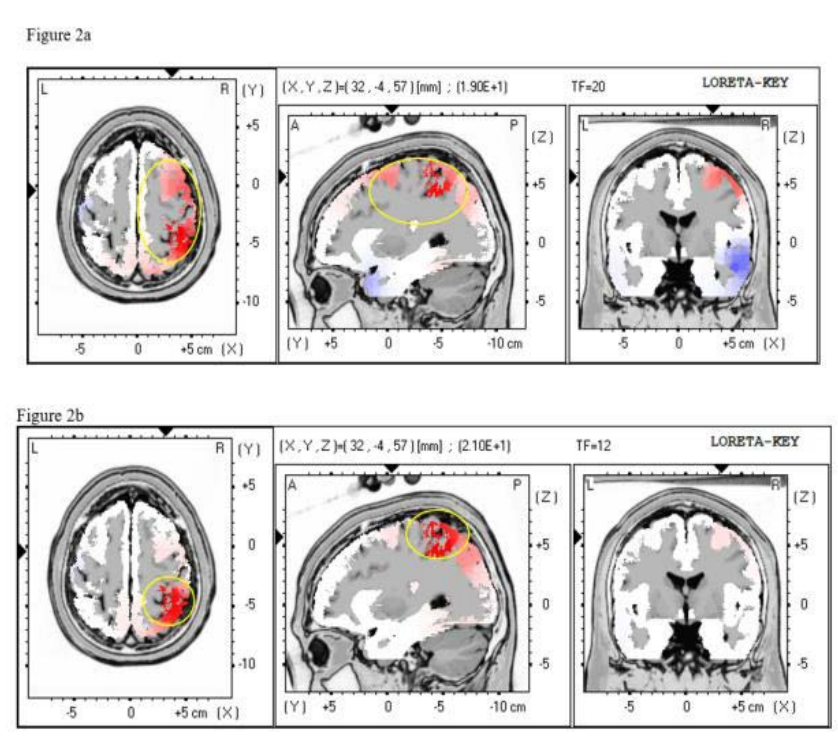

Figures 2a \& 2b: Placebo: Beta $(20 \mathrm{HZ})$ at BA 6 increased from pre- to postplacebo treatment (Figure 2a) and alpha also increased (Figure 2b).

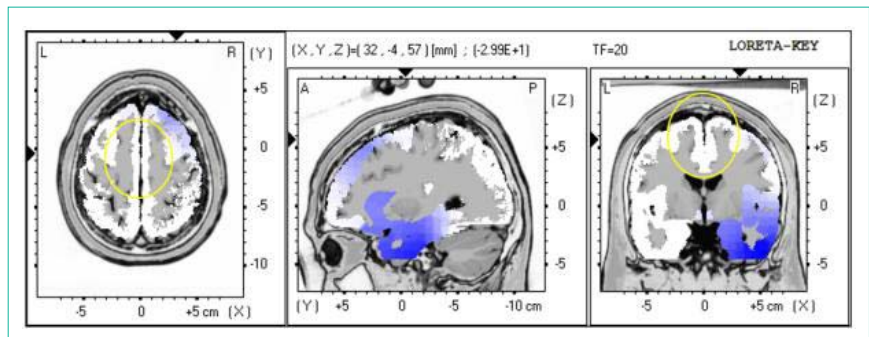

Figure 3: Waitlist: Beta $(2 \mathrm{OHZ})$ at BA 6 did not change during time on waitlist.

with work, walking, sleep, mood, and overall enjoyment of life. The patient reported taking ibuprofen to control CIPN symptoms. After 20 treatment sessions, the patient rated her neuropathy as a $0 / 10$ in both hands and feet. She reported regained tactile sensation, ability to feel temperature and texture on toes and fingers, ability to dance and walk without being limited by neuropathy and feeling more relaxed. After 20 PL sessions, the patient's EEG showed an increase in beta frequencies associated with pain-related brain activity (Figure 2a) and a widespread increase in alpha frequencies (Figure $2 b$ ).

While receiving the elective NFB sessions, the patient reported improvement in numbness which allowed her to "actually do the craft things [she] couldn't do before" such as hanging shelves in her home. After 14 sessions, she reported that she could "feel [her] cat's fur ... for the first time since before chemo." At the conclusion of 20 elective NFB sessions, she rated her neuropathy as a $1 / 10$ in her hands and $0 / 10$ in her feet. She showed brain changes that were not targeted by the protocols.

\section{Case 3: Waitlist followed by elective NFB}

A 73-year-old female diagnosed with Stage II breast cancer who reported experiencing CIPN for 9 months at the time of consent and self-reported an overall neuropathy rating of $3 / 10$. The patient reported moderate neuropathic pain and heightened skin sensitivity to heat that interfered with her ability to carry out daily hygiene and household responsibilities. She often found it necessary to 
wear gloves while washing dishes or driving but found this did not completely alleviate neuropathic symptoms. The patient reported that heightened pain led to diminished energy, noting that on some days she "just wanted to stay on the couch." To control symptoms, she reported taking Ibuprofen and T-relief (an over-the-counter natural pain reliever) as needed, as well as underwent acupuncture and chiropractic sessions.

There was no change in neuronal oscillations at BA6 from baseline measurement to the end of the 10-week waiting period (Figure 3). The patient's neuropathy rating after 10 weeks had slightly worsened to a $4 / 10$.

After the follow-up assessment, the patient elected to receive real NFB. She experienced increased energy "almost immediately" after beginning NFB sessions. After 20 sessions, she reported decreased fatigue, continued increased energy, and a reduced neuropathy rating of 1/10. She did not have any change in brain activity in BA 6 from pre-to-post treatment.

\section{Discussion}

A conundrum in clinical work has been accurately linking patient reported outcomes with objective measures, and further to identify mechanisms of interventional trials. At the conclusion of the parent trial (randomized, placebo-controlled) we selected three patients who were assigned to different intervention groups. The two patients in the treatment groups improved as measured by self-report, but their brain data varied according to treatment received.

The PL participant had an improvement in CIPN symptoms, with increases in beta and alpha activity $(12 \mathrm{~Hz})$. This increase in alpha may have contributed to the participant's symptomatic improvement Placebo studies demonstrate increases in alpha activation in the brain during placebo analgesia and have suggested that augmenting alpha may decrease perception of pain [4]. Rather than a "false" improvement, placebo may be conceived of as a normal, active mechanism in medical interventions, though its effect on the body may be different from that of targeted interventions. Though both the NFB and PL patients reported similar symptom improvements, EEG data suggests divergent drivers of therapeutic gains. The changes achieved over the course of NFB were consistent with the targeted NFB training goals, while changes for PL appear to reflect more general placebo analgesia mechanisms. The EEG signature of the WL participant did not significantly change over the course of the waiting period.

\section{Conclusion}

These cases provide insight into the effects of CIPN on the daily lives of cancer survivors, highlight the benefits of pursuing treatment strategies such as NFB, and suggest differential EEG changes associated with improved symptoms for NFB and PL treatments. Decreasing beta may lead to symptom improvement, as our previous research suggests; however, the experience of PL responders (and the evidence of post-PL EEG changes) suggest it is also possible to achieve symptomatic relief by other neuromodulatory routes. Our intention is not to draw the conclusion that all patients in the three randomized groups will have similar brain data as these cases, but rather to propose that unity between objective and subjective measures can achieved.

\section{Disclosures and Acknowledgments}

Funding: This work was supported by the National Center for Complementary and Integrative Health [grant number K01AT008485] and the Rising Tide Foundation [grant number CCR14-800].

The authors would like to thank the Foundation for Neurofeedback \& Applied Neuroscience and EEGer for the donation of neurofeedback equipment and software used to conduct this study.

\section{References}

1. Dizon DS, Krilov L, Cohen E, Gangadhar T, Ganz PA, Hesing TA, et al. Clinical Cancer Advances 2016: Annual Report on Progress Against Cancer From the American Society of Clinical Oncology. Journal of Clinical Oncology. 2016; 34: 987-1011.

2. Seretny M, Currie GL, Sena ES, Ramnarine S, Grant R, Macleod MR, et al. Incidence, prevalence, and predictors of chemotherapy-induced peripheral neuropathy: a systematic review and meta-analysis. PAIN. 2014; 155: $2461-$ 2470.

3. Prinsloo S, Novy D, Driver L, Lyle R, Ramondetta L, Eng C, et al. Randomized controlled trial of neurofeedback on chemotherapy-induced peripheral neuropathy: A pilot study. Cancer. 2017; 123: 1989-1997.

4. Huneke NT, Brown CA, Burford E, Watson A, Trujillo-Barreto NJ, Ei-Deredy $\mathrm{W}$, et al. Experimental placebo analgesia changes resting-state alpha oscillations. PLoS One. 2013; 8: e78278. 